\title{
Absence of Weak Local Rules for the Planar Quasicrystalline Tiling with the 8-fold Rotational Symmetry
}

\author{
S. E. Burkov
}

Landau Institute for Theoretical Physics, Moscow, USSR

\begin{abstract}
Levitov's theory of local rules (the preceding paper of this issue) gives no prediction for the planar quasicrystalline tiling having 8-fold rotational symmetry. Absence of weak local rules for this particular tiling is proven.
\end{abstract}

A general theory of local rules for planar tilings has been developed by Levitov [1]. Below we study one of the simplest particular cases: a planar tiling with the 8-fold symmetry. This tiling can be obtained by projecting from the four-dimensional space. Matrix elements of the projector contain only one irrational number $\sqrt{2}$, which is a quadratic irrationality. Nevertheless, Levitov's Theorem 3 which states that quadratic irrationalities give rise to tilings with at least weak local rules, cannot be applied to our particular case. The theorem can be applied only to the tilings with the non-degenerate SI-conditions [1]. It is easy to show that in our case the SI-conditions are degenerate. Specific properties of the continued fraction $\sqrt{2}$ $=\{1,2,2,2,2 \ldots\}$ along with the four-dimensionality of the total space and the 8 -fold symmetry of the tiling enable one to prove the absence of weak local rules directly, without use of the general theory. Evidence for the absence of strong local rules for this particular tiling was given by Beenker [2]. Absence of strong local rules does not result in absence of weak rules, but absence of weak rules results in absence of strong rules. Thus, Beenker's statement is a consequence of the assertion proven below.

Definitions of tiles, tilings, quasicrystalline tilings, the lattice surface in total space, strong and weak local rules are given in the Levitov's paper [1]. We shall not give them once again. Define the particular tiling with the 8 -fold symmetry. The tiling is quasicrystalline, i.e. it can be treated as a projection of the 2- $D$ lattice surface confined in the standard tube onto some $2-D$ subspace $\mathbb{R}_{\sqrt{2}}^{2} \subset \mathbb{R}^{4}[1,3]$. We set this subspace by fixing the projections of unit basic vectors of $\mathbb{R}^{4}$ onto $\mathbb{R}_{\sqrt{2}}^{2}$ :

$$
\begin{array}{ll}
\mathbf{e}_{1}^{\prime}=\frac{1}{2}\left(1 ; \frac{\sqrt{2}}{2} ; 0 ;-\frac{\sqrt{2}}{2}\right), \quad\left|\mathbf{e}_{j}^{\prime}\right|=\frac{\sqrt{2}}{2} \\
\mathbf{e}_{2}^{\prime}=\frac{1}{2}\left(\frac{\sqrt{2}}{2} ; 1 ; \frac{\sqrt{2}}{2} ; 0\right),
\end{array}
$$




$$
\begin{aligned}
& \mathbf{e}_{3}^{\prime}=\frac{1}{2}\left(0 ; \frac{\sqrt{2}}{2} ; 1 ;-\frac{\sqrt{2}}{2}\right), \\
& \mathbf{e}_{4}^{\prime}=\frac{1}{2}\left(-\frac{\sqrt{2}}{2} ; 0 ; \frac{\sqrt{2}}{2} ; 1\right) .
\end{aligned}
$$

Following Gahler and Rhyner [4] we shall call $\mathbb{R}_{\sqrt{2}}^{2}$ a tiling plane, $\mathbf{e}_{j}^{\prime}$ - tiling vectors. The four tiling vectors $\mathbf{e}_{j}^{\prime}$ are shown in Fig. $1 \mathrm{a}$. The symmetry reduces the number of tile types from $\left(\begin{array}{l}2 \\ 4\end{array}\right)=6$ to 2 : tiles $\langle 1,3\rangle$ and $\langle 2,4\rangle$ are squares, tiles $\langle 1,2\rangle$, $\langle 2,3\rangle,\langle 3,4\rangle$, and $\langle 1,4\rangle$ are rhombi of the $45^{\circ}$ angle. Equation (1) defines a family of quasicrystalline tilings, each tiling corresponding to some position of the tube in $\mathbb{R}^{4}$. We shall fix the position later. The tiling itself is shown in Fig. 2.

Define a one-parameter family $\left\{\mathscr{T}_{w}\right\}$ of auxiliary tilings of the same tiling plane $\mathbb{R}_{\sqrt{2}}^{2}$ by the same tiles (a square and a $45^{\circ}$ rhombus). Introduce a family of the $2-D$

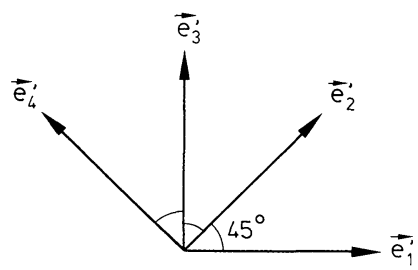

a

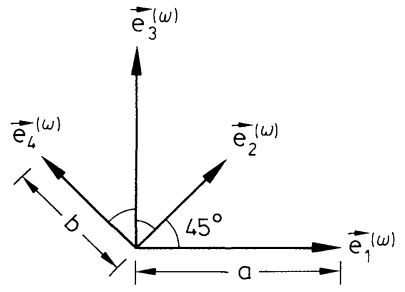

b

Fig. 1 a and b. Projections of the unit basic vectors onto the tiling plane $\mathbb{R}_{V \overline{2}}^{2} \mathbf{a}$ and onto the grid plane $\mathbb{R}_{w}^{2} \mathbf{b}$
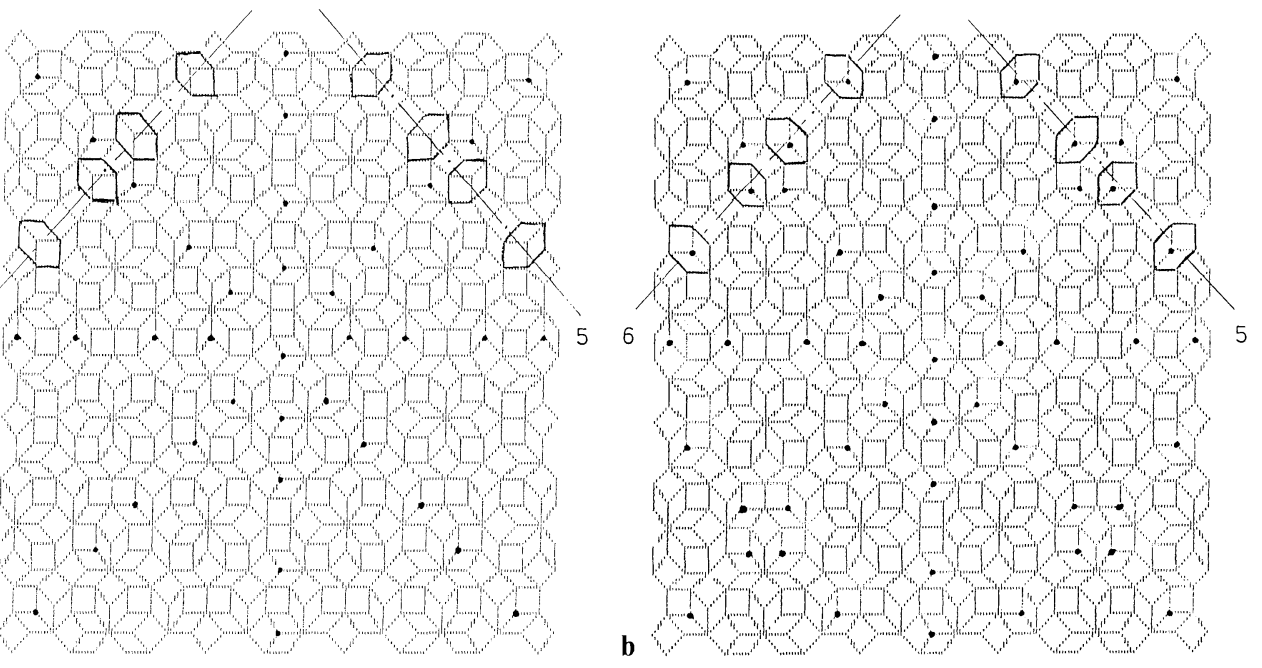

Fig. 2a and b. Fragments of the quasicrystalline tiling with the 8-fold symmetry $\mathscr{T}_{\sqrt{2}}$ a and of the period tiling $\mathscr{T}_{w_{k}}\left(w_{2}=7 / 5\right) \mathbf{b}$. Diophantine points are shown by solid circles. The two tilings coincide except for 8 perestroikas located on lines 5 and 6 (dashed) 
subspace $\mathbb{R}_{w}^{2}, w \in \mathbb{R}^{1}$, called grid spaces. Define $\mathbb{R}_{w}^{2}$, as in Eq. (1), by fixing the projections of the unit basic vectors onto $\mathbb{R}_{w}^{2}$ :

$$
\begin{array}{ll}
\mathbf{e}_{1}^{(w)}=a^{2}\left(1 ; \frac{1}{w} ; 0 ;-\frac{1}{w}\right), & \left|\mathbf{e}_{1}^{(w)}\right|=a, \\
\mathbf{e}_{2}^{(w)}=b^{2}\left(\frac{w}{2} ; 1 ; \frac{w}{2} ; 0\right), & \left|\mathbf{e}_{2}^{(w)}\right|=b, \\
\mathbf{e}_{3}^{(w)}=a^{2}\left(0 ; \frac{1}{w} ; 1 ; \frac{1}{w}\right), & \left|\mathbf{e}_{3}^{(w)}\right|=a, \\
\mathbf{e}_{4}^{(w)}=b^{2}\left(-\frac{w}{2} ; 0 ; \frac{w}{2} ; 1\right), & \left|\mathbf{e}_{4}^{(w)}\right|=b, \\
a^{2}=1 /\left(1+2 / w^{2}\right), & b^{2}=1 /\left(1+w^{2} / 2\right) .
\end{array}
$$

The vectors $\mathbf{e}_{j}^{\prime}$ are shown in Fig. 1 b. The tiling plane $\mathbb{R}_{\sqrt{2}}^{2}$ is a member of the family $\mathbb{R}_{w}^{2}$ corresponding to $w=\sqrt{2}$. Consider a standard tube $\Pi_{w}$ which is parallel to $\mathbb{R}_{w}^{2}$ and project a $2-D$ lattice surface confined in it onto $\mathbb{R}_{\sqrt{2}}^{2}$. Thus, for every $w \in \mathbb{R}^{1}$ we have some tiling of the tiling plane $\mathbb{R}_{\sqrt{2}}^{2}$ (the position of the tube in $\mathbb{R}^{4}$ will be fixed later). The tiling $\mathscr{T}_{w}$ is quasiperiodic if $w$ is irrational, it is periodic if $w=p / q(p, q \in \mathbb{Z})$. The periods are:

$$
\begin{gathered}
\mathbf{T}_{1}=p \mathbf{e}_{1}^{\prime}+q \mathbf{e}_{2}^{\prime}-q \mathbf{e}_{4}^{\prime}, \\
\mathbf{T}_{1}=\quad q \mathbf{e}_{2}^{\prime}+p \mathbf{e}_{3}^{\prime}-q \mathbf{e}_{4}^{\prime}, \\
\left|\mathbf{T}_{1}\right|=\left|\mathbf{T}_{2}\right|=T, \quad \mathbf{T}_{1} \cdot \mathbf{T}_{2}=0,
\end{gathered}
$$

i.e. the tiling can be considered as a crystal with the square lattice (Fig. 5).

Consider the 2-D subspaces $\mathbb{R}_{w}^{2 *}$ which are orthogonal to $\mathbb{R}_{w}^{2}[3,4]$. Take some $w \in \mathbb{R}^{1}$ and project onto $\mathbb{R}_{w}^{2 *}$ all the points $\mathbf{X} \in \mathbb{Z}^{4}$ lying in the tube $\Pi_{w}$ (recall that these points are vertices of the $2-D$ lattice surface generating the tiling). Projections of all these $\mathbf{X} \in \mathbb{Z}^{4}$ onto $\mathbb{R}_{w}^{2 *}$ are confined in the octagon, which, in turn, is the projection of the unit 4-D cube onto $\mathbb{R}_{w}^{2 *}[3,4]$. The shift of the tube in $\mathbb{R}^{4}$ results in the shift of the octagon in $\mathbb{R}_{w}^{2 *}$. Now we fix the position of the tube $\Pi_{w}$ :

$$
\begin{array}{lll}
-2 \leqq \vec{N}_{1}^{(w)} \cdot \vec{X}<\frac{2}{w}, & \vec{N}_{1}=\left(0 ;-1 ; \frac{2}{w} ;-1\right), & \left|\vec{N}_{1}\right|=\sqrt{2} / a, \\
-1<\vec{N}_{2}^{(w)} \cdot \vec{X} \leqq 1+w, & \vec{N}_{2}=(1 ; 0 ;-1 ; w), & \left|\vec{N}_{2}\right|=\sqrt{2} / b, \\
-1 \leqq \vec{N}_{3}^{(w)} \cdot \vec{X}<1+\frac{2}{w}, & \vec{N}_{3}=\left(\frac{2}{w} ;-1 ; 0 ; 1\right), & \left|\vec{N}_{3}\right|=\sqrt{2} / a, \\
-2<\vec{N}_{4}^{(w)} \cdot \vec{X} \leqq w, & \vec{N}_{4}=(-1 ; w ;-1 ; 0), & \left|\vec{N}_{4}\right|=\sqrt{2} / b,
\end{array}
$$

where $\mathbf{N}_{j}^{(w)} \in \mathbb{R}_{w}^{2 *} \subset \mathbb{R}^{4}$ are the vectors perpendicular to the sides of the octagon (Fig. 3). Strictly speaking, although Eq. (4) defines the tiling unambiguously, it is not consistent with Levitov's definition of the quasicrystalline tiling [1]. We have chosen the position of the tube so that there are points $\mathbf{X} \in \mathbb{Z}^{4}$ belonging to the 


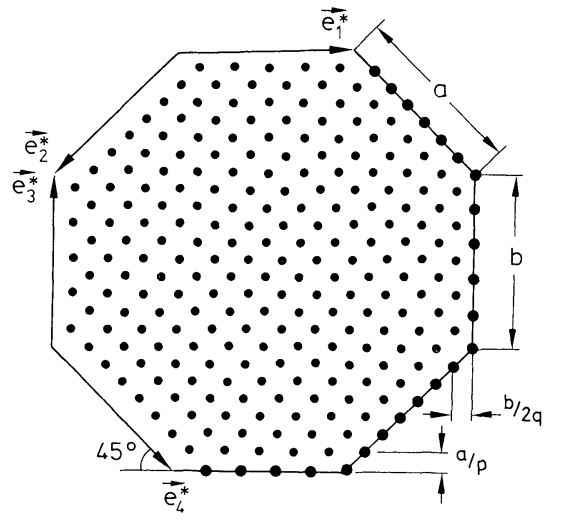

Fig. 3. Projection of the standard tube $\Pi_{w_{2}}$ onto $\mathbb{R}_{w_{2}}^{2 *}\left(w_{2}=p / q=7 / 5\right)$. Projections of the Diophantine points are shown by solid circles

surface of the tube. It is forbidden by Levitov's definition. Thus, our definition of the quasicrystalline tiling is slightly more general: the tube may intersect $\mathbb{Z}^{4}$, but in this case some strong inequalities in equations analogous to Eq. (4) should be replaced by weak inequalities. It can be shown that such generalization is no problem. The proof can be repeated just in terms of Levitov's definitions, but Eq. (4) should be modified by the infinitesimal tube shift:

$$
-2+\varepsilon_{1}<\vec{N}_{1}^{(w)} \cdot \vec{X}<\frac{2}{w}+\varepsilon_{1},
$$

etc., where $\varepsilon_{1}$ can be arbitrarily small. We shall not give here these $\varepsilon_{1}$ but generalize the definition and put $\varepsilon_{1}=0$. We shall call the points $\mathbf{X} \in \mathbb{Z}^{4}$ lying on the tube surface Diophantine. Their projections onto $\mathbb{R}_{w}^{2 *}$ lie on the sides of the octagon. The Diophantine points projected onto the side $j$ will be called points of type $j$ $(j=1,2,3,4$, see Fig. 3). For example, the Diophantine points of type 1 are the solutions of the Diophantine equation

$$
\vec{N}_{1}^{(w)} \cdot \vec{X}=-2, \quad \mathbf{X} \in \mathbb{Z}^{4},
$$

satisfying three other inequalities (4). If $w$ is irrational the solution is:

$$
\begin{gathered}
\mathbf{X}=\operatorname{Int}\left(n \mathbf{A}_{1}\right)+\mathbf{e}_{2}+\mathbf{e}_{4}, \quad n \in \mathbb{Z}, \\
\mathbf{A}_{1}=\left(w+\frac{2}{w}\right) \mathbf{e}_{1}^{(w)}=(w, 1,0,-1) \in \mathbb{R}_{w}^{2},
\end{gathered}
$$

where $\operatorname{Int}\left(n \mathbf{A}_{1}\right)=(\operatorname{Int}(n w) ; n ; 0 ;-n) \in \mathbb{Z}^{4}$.

Projections of the Diophantine points (6) onto $\mathbb{R}_{w}^{2 *}$ densely fill side 1 of the octagon:

$$
\mathbf{X}^{*}=\mathbf{e}_{2}^{*}+\mathbf{e}_{4}^{*}-\operatorname{Frac}(n w) \mathbf{e}_{1}^{*}, \quad n \in \mathbb{Z} .
$$

Their projections onto the tiling plane $\mathbb{R}_{\sqrt{2}}^{2}$ are situated along the straight line which is parallel to $\mathbf{e}_{1}^{\prime}$ :

$$
\begin{aligned}
\mathbf{X}^{\prime} & =\mathbf{e}_{2}^{\prime}+\mathbf{e}_{4}^{\prime}+n \mathbf{A}_{1}^{\prime}-\operatorname{Frac}(n w) \mathbf{e}_{1}^{\prime}=\mathbf{e}_{2}^{\prime}+\mathbf{e}_{4}^{\prime}+\operatorname{Int}(n w) \mathbf{e}_{1}^{\prime}+n \mathbf{e}_{2}^{\prime}-n \mathbf{e}_{4}^{\prime} \\
& =\mathbf{e}_{2}^{\prime}+\mathbf{e}_{4}^{\prime}+(\operatorname{Int}(n w)+n \sqrt{2}) \mathbf{e}_{1}^{\prime} .
\end{aligned}
$$




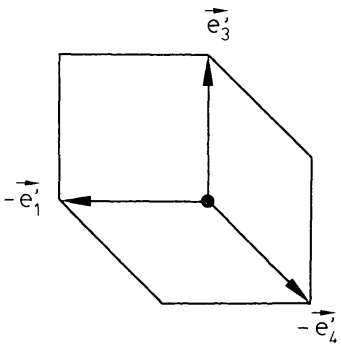

a

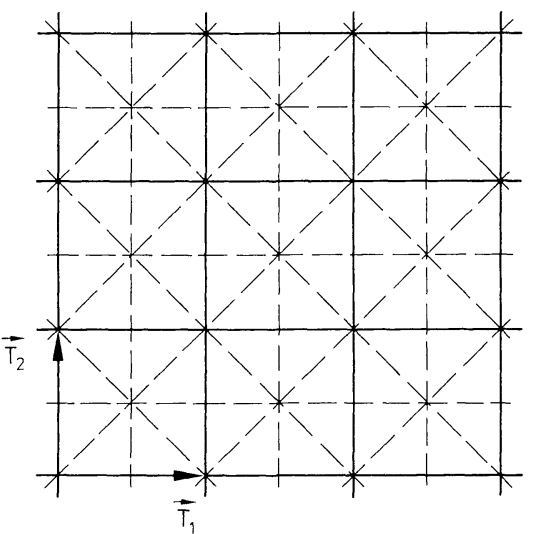

$\overrightarrow{\mathrm{e}_{4}}$

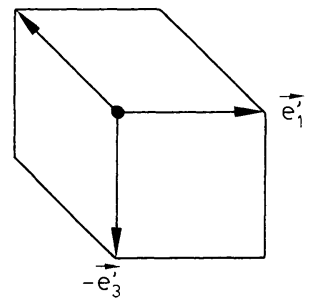

b

Fig. 4. a Three tiles surrounding the Diophantine points of type 2 [which satisfy the equation $\vec{N}_{2} \cdot \vec{X}=1+w$, Eq. (4.1)]. $b$ Permutation of the three tiles, called perestroika, corresponds to the change $\mathbf{X} \rightarrow \mathbf{X}-\mathbf{e}_{1}+\mathbf{e}_{3}-\mathbf{e}_{4}$ (Lemma 3). New $\mathbf{X}$ satisfies the equation $\vec{N}_{2} \cdot \vec{X}=-1$

Fig. 5. Diophantine points of the periodic tiling $\mathscr{T}_{w_{k}}$ are situated on the dashed lines. Solid lines are the boundaries of the elementary cells. 9 cells are shown $(-1.5 T<X<1.5 T,-1.5 T<Y<1.5 T)$

A fragment of the tiling near the Diophantine point of type 2 is shown in Fig. 4. The Diophantine points of types 3, 4 are found analogously. Thus, in the case of irrational $w$ the projections of the Diophantine points onto the tiling plane are situated along four straight lines which are parallel to the tiling vectors $\mathbf{e}_{j}^{\prime}$. In the case of rational $w$, Eqs. (6)-(8) give the positions of the Diophantine points within the elementary cell, which is a square with the sides $\mathbf{T}_{1}, \mathbf{T}_{2}[$ Eq. (3)]. Since the tiling is periodic with the periods $\mathbf{T}_{1}, \mathbf{T}_{2}$, all the Diophantine points are situated on the lines shown in Fig. 5.

Theorem. For the quasicrystalline tiling $\mathscr{T}_{\sqrt{2}}$ obtained by projecting the standard tube which is parallel to the plane $\mathbb{R}_{\sqrt{2}}^{2}$ (Eq. (1)) onto the same plane $\mathbb{R}_{\sqrt{2}}^{2}$, there are no weak local rules of any radius.

Let us suppose that there exist rules of the radius $R$. Let $w_{k}=p_{k} / q_{k}$ be a rational approximant of $\sqrt{2}$ obtained by the truncation of the continued fraction: $w_{k}=\{1,2,2, \ldots, 2\}$ (the element 2 is repeated $k$ times). Take $k$ large enough, so that, say, $q_{k}>100 R$. Compare a periodic tiling $\mathscr{T}_{w_{k}}$ associated with this $w_{k}$ and the given quasiperiodic tiling $\mathscr{T}_{\sqrt{2}}$ associated with $w=\sqrt{2}$. We intend to prove that the periodic tiling comprises allowed configurations only. Thus, we must prove that any fragment of the periodic tiling confined in a circle of the radius $R$ can be found in the given quasiperiodic tiling. The periodicity allows us to consider not all the periodic tiling $\mathscr{T}_{w_{k}}$, but its final portion, namely a square consisting of one period plus a frame of the width $2 R$ (Fig. 6). We consider even a larger square, namely a square with the sides $2 \mathbf{T}_{1}, 2 \mathbf{T}_{2}$ (Fig. 6). 


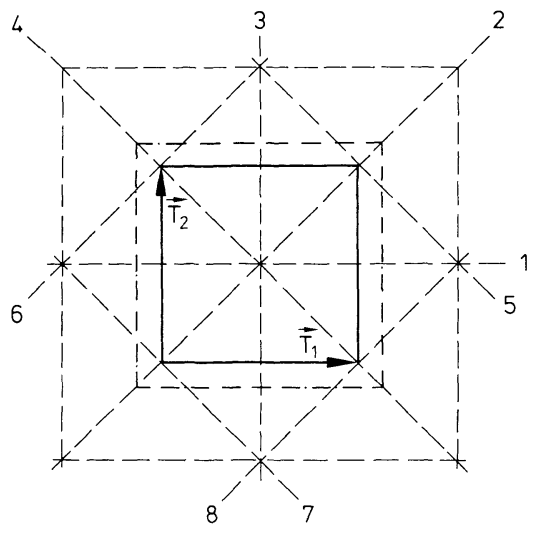

Fig. 6. The square $-T<X<T,-T<Y<T$ used in the proof. The elementary cell is shown by the solid line. The square shown by the dashed-dotted line is larger than the elementary cell by the value $4 R$ ( $R$ is the rules radius). The Diophantine points of $\mathscr{T}_{w_{k}}$ are located on the dashed lines (the boundaries of this square are not used in the proof)

Lemma 1. For large enough $k$,

$$
p_{k}-\sqrt{2} q_{k} \simeq \frac{(-1)^{k+1}}{2 \sqrt{2}} \cdot \frac{1}{q_{k}} .
$$
$[5]$ :

Since $\sqrt{2}=\{1,2,2, \ldots, 2, \ldots\}, p_{k}$ and $q_{k}$ are defined by the recurrent procedure

$$
\begin{array}{ll}
p_{k+1}=2 p_{k}+p_{k-1}, & p_{0}=1, p_{1}=3, \\
q_{k+1}=2 q_{k}+q_{k-1}, & q_{0}=1, q_{1}=2 .
\end{array}
$$

It is easy to find that

$$
\begin{aligned}
& p_{k}=\frac{1}{2} \cdot\left(\lambda_{1}^{k+1}+\lambda_{2}^{k+1}\right), \\
& q_{k}=\frac{1}{2 \sqrt{2}}\left(\lambda_{1}^{k+1}-\lambda_{2}^{k+1}\right), \\
& p_{k}-\sqrt{2} q_{k}=\lambda_{2}^{k+1},
\end{aligned}
$$

where

$$
\begin{aligned}
& \lambda_{1}=1+\sqrt{2} \approx 2.4, \\
& \lambda_{2}=1-\sqrt{2} \approx-0.4
\end{aligned}
$$

are the eigenvalues of the matrix

$$
\left(\begin{array}{ll}
2 & 1 \\
1 & 0
\end{array}\right)
$$

For the large enough $k$, Eq. (10.2) can be written as $q_{k} \simeq \lambda_{1}^{k+1} / 2 \sqrt{2}$. Combining it with Eq. (10.3) we have Eq. (9).

Lemma 2. The tilings $\mathscr{T}_{w_{k}}$ and $\mathscr{T}_{\sqrt{2}}$ coincide within the square $-T<x<T$, $-T<y<T$ (Fig. 6) except, may be, in the Diophantine points of the periodic tiling $\mathscr{T}_{w_{k}}$. 
To prove Lemma 2 we should prove that if any $\mathbf{X} \in \mathbb{Z}^{4}$ is such that:

- $\mathbf{X}$ belongs the standard tube $\Pi_{w_{k}}$

- $\mathbf{X}$ is not a Diophantine point of $\mathscr{T}_{w_{k}}$

- the projection of $\mathbf{X}$ onto the tiling plane belongs the square in Fig. 6, then $\mathbf{X}$ belongs the standard tube $\Pi_{\sqrt{2}}$.

Since $\mathbf{X} \in \Pi_{w_{k}}$, inequality (4.1) is fulfilled:

$$
-2 \leqq \vec{N}_{1}^{\left(w_{k}\right)} \cdot \vec{X} \leqq \frac{2 q_{k}}{p_{k}} .
$$

Since $\mathbf{X}$ is not a Diophantine point of type 1, the minimal distance between $\mathbf{X}$ and the surface of the tube $\Pi_{w_{k}}$ is $b^{\left(w_{k}\right)} / 2 q$ (sides 1 and 3 have the length $b^{\left(w_{k}\right)}$, there are $q$ Diophantine points on each side as is shown in Fig. 3). This fact means that inequality (11) becomes stronger:

$$
-2+\frac{1}{p_{k}} \leqq \vec{N}_{1}^{\left(w_{k}\right)} \cdot \vec{X} \leqq \frac{2 q_{k}}{p_{k}}-\frac{1}{p_{k}} .
$$

Calculate $\vec{N}_{1}^{(\sqrt{2})} \cdot \vec{X}-\vec{N}_{1}^{\left(w_{k}\right)} \cdot \vec{X}$ :

$$
\left(\vec{N}_{1}^{(\sqrt{2})}-\vec{N}_{1}^{\left(w_{k}\right)}\right) \cdot \vec{X}=\left(0 ; \frac{2}{\sqrt{2}} ; \frac{2}{w_{k}} ; 0\right) \cdot \vec{X}=\frac{2\left(p_{k}-\sqrt{2} q_{k}\right) X_{3}}{\sqrt{2} p_{k}} .
$$

Substituting Eq. (9) we have

$$
\vec{N}_{1}^{(\sqrt{2})} \cdot \vec{X}-\vec{N}_{1}^{\left(w_{k}\right)} \cdot \vec{X}=(-1)^{k+1} \frac{\sqrt{2}}{2} \cdot \frac{X_{3}}{p_{k}^{2}}
$$

The fact that $\mathbf{X}$ is projected onto the square in Fig. 6 means that

$$
-p<X_{1}<p, \quad-2 q<X_{2}<2 q, \quad-p<X_{3}<p, \quad-2 q<X_{4}<2 q .
$$

Therefore

$$
\left|\vec{N}_{1}^{(\sqrt{2})} \cdot \vec{X}-\vec{N}_{1}^{\left(w_{k}\right)} \cdot \vec{X}\right|<\frac{2}{\sqrt{2}} \cdot \frac{1}{p_{k}} .
$$

Bearing in mind that $\left|\frac{2 q_{k}}{p_{k}}-\sqrt{2}\right| \sim \frac{1}{p_{k}^{2}} \ll \frac{1}{p_{k}}$, Eqs. (12) and (15) yield:

$$
-2<\vec{N}_{1}^{(\sqrt{2})} \cdot \vec{X}<\sqrt{2} .
$$

Equation (16) is just the first inequality (13) for $w=\sqrt{2}$. The three other inequalities can be derived analogously.

Lemma 3. The tilings $\mathscr{T}_{w_{k}}$ and $\mathscr{T}_{\sqrt{2}}$ coincide in the Diophantine points of the $\mathscr{T}_{w_{k}}$ lying on lines 1,2,3,4 in Fig. 6 . If $k$ is odd they coincide on lines 5,6 and do not coincide on lines 7, 8 ; if $k$ is even then they coincide on lines 7,8 and do not coincide on lines 5,6 .

We prove that if $\mathbf{X} \in \mathbb{Z}^{4}$ is a Diophantine point of type 1 of the periodic tiling $\mathscr{T}_{w_{k}}$ it belongs the tube $\Pi_{\sqrt{2}}$, moreover it is also a Diophantine point of the quasiperiodic tiling $\mathscr{T}_{\sqrt{2}}$. According to Eq. (6): 


$$
\mathbf{X}=\left(\operatorname{Int}\left(n w_{k}\right) ; n ; 0 ;-n\right)+(0 ; 1 ; 0 ; 1)
$$

hence

$$
\vec{N}_{1}^{(\sqrt{2})} \cdot \vec{X}=(0,-1, \sqrt{2},-1) \cdot \vec{X}=-2 .
$$

It is easy to check that when $\mathbf{X} \in \Pi_{w_{k}}$, it satisfies the three other inequalities (4). Thus, the tiling $\mathscr{T}_{w_{k}}$ and $\mathscr{T}_{\sqrt{2}}$ coincide in the Diophantine points of type 1, which are situated on line 1 . The proof for lines 2,3,4 is analogous.

The Diophantine points of $\mathscr{T}_{w_{k}}$ lying on lines 6 and 8 respectively are:

$$
\begin{aligned}
& \mathbf{X}=\left(n ; \operatorname{Int}\left(\frac{2 n}{w_{k}}\right) ; n ; 0\right)+(0 ; q, p, q)+(1,0,0,1), \\
& \mathbf{X}=\left(n ; \operatorname{Int}\left(\frac{2 n}{w_{k}}\right) ; n ; 0\right)-(0 ; q, p, q)+(1,0,0,1)
\end{aligned}
$$

[they are of type 2 , the projection of $(0, q, p, q)$ onto the tiling plane is $\mathbf{T}_{2}$, Eq. (3)]. It is easy to see that inequalities (4.1), (4.3), (4.4) are fulfilled. Inequality (4.2) should be carefully checked. For the line 6 we have:

$$
\vec{N}_{2}^{(\sqrt{2})} \cdot \vec{X}=a+(1,0,-1, \sqrt{2}) \cdot \vec{X}=1+\sqrt{2}+\sqrt{2} q_{k}-p_{k}=1+\sqrt{2}+(-1)^{k}\left|\lambda_{2}\right|^{k+1} .
$$

If $k$ is even then

$$
\vec{N}_{2}^{(\sqrt{2})} \cdot \vec{X}=1+\sqrt{2}+\left|\lambda_{2}\right|^{k+1}>1+\sqrt{2},
$$

i.e. the Diophantine points of the periodic tiling $\mathscr{T}_{w_{k}}$, which lie on line 6 , do not belong the tube $\Pi_{\sqrt{2}}$. If we add $-\mathbf{e}_{1}+\mathbf{e}_{3}-\mathbf{e}_{4}$ to these $\mathbf{X}$ we obtain

$$
\begin{gathered}
\mathbf{X}^{\dagger}=\left(n ; \operatorname{Int}\left(\frac{2 n}{w_{k}}\right) ; n ; 0\right)+(0, q, p, q)+(0,0,1,0), \\
\vec{N}_{2}^{(\sqrt{2})} \cdot \vec{X}^{\dagger}=-1+\left|\lambda_{2}\right|^{k+1}>-1,
\end{gathered}
$$

i.e. $\mathbf{X}^{\dagger}$ belong the tube $\Pi_{\sqrt{2}}$. Thus, the tilings $\mathscr{T}_{w_{k}}$ and $\mathscr{T}_{\sqrt{2}}$ do not coincide in the Diophantine points lying on line 6 . They differ by perestroikas in these points (Figs. 3, 4).

For line 8 we have:

$$
\vec{N}_{2}^{(\sqrt{2})} \cdot \vec{X}=-\left(\sqrt{2} q_{k}-p_{k}+1+\sqrt{2}\right)=1+\sqrt{2}+(-1)^{k+1}\left|\lambda_{2}\right|^{k+1} .
$$

If $k$ is even then

$$
\vec{N}_{2}^{(\sqrt{2})} \cdot \vec{X}=1+\sqrt{2}-\left|\lambda_{2}\right|^{k+1}
$$

i.e. $\mathbf{X}$ belongs $\Pi_{\sqrt{2}}$ and, therefore, the two tilings coincide on line 8 . If $k$ is odd lines 6 and 8 are permuted. The proof for lines 5 and 7 is analogous.

Proof of the Theorem. Lemmas 2, 3 show that the tiling $\mathscr{T}_{w_{k}}$ and $\mathscr{T}_{\sqrt{2}}$ coincide within our square (Fig. 6) except a finite number of perestroikas which are located on lines 5 and 6 (let $k$ be even). Consider an arbitrary circle of the radius $R$ which is situated in the square $-2 R-\frac{T}{2}<X<\frac{T}{2}+2 R,-2 R-\frac{T}{2}<Y<\frac{T}{2}+2 R$, (it is 
shown by a dashed-dotted line in Fig. 6). If this circle does not intersect lines 5, 6, then the fragment of the tiling $\widetilde{T}_{w_{k}}$ confined by this circle is identical to those of the given quasicrystalline tiling $\mathscr{T}_{\sqrt{2}}$. If the circle intersects lines 5 or 6 , then we should consider another circle shifted by $-\mathbf{T}_{2}$ with respect to the initial circle. Since $\mathbf{T}_{2}$ is a period of the tiling $\mathscr{T}_{w_{k}}$, the fragments of $\mathscr{T}_{w_{k}}$ confined by both circles are identical. The new circle does not intersect lines 5,6 , hence, the fragment of $\mathscr{T}_{w_{k}}$ in it coincides with the fragment of $\mathscr{T}_{\sqrt{2}}$. Thus, the fragment of the tiling $\mathscr{T}_{w_{k}}$ in the given circle is identical to the fragment of the tiling $\mathscr{T}_{\sqrt{2}}$ in the shifted circle. We see that the fragment of the periodic tiling $\mathscr{T}_{w_{k}}$ confined by any circle of radius $R$ can be found among the allowed configurations (i.e. configurations of tiles confined in the circle of the radius $R$ which are fragments of the given quasicrystalline tiling $\mathscr{T}_{\sqrt{2}}$ ). So, allowed configurations may form not only the given tiling $\widetilde{T}_{\sqrt{2}}$ but at least another tiling $\mathscr{T}_{w_{k}}$. According to Levitov's definition it means that the quasicrystalline tiling $\mathscr{T}_{\sqrt{2}}$ has no weak local rules of any radius $R$.

Acknowledgements. The author is indebted to L.S. Levitov for informing him about his local rule theory prior to its publication, as well as for many useful discussions and answering an enormous number of questions concerning that theory. The author is thankful to Ya. G. Sinai for his interest in the work.

\section{References}

1. Levitov, L.S.: The preceding paper of this issue

2. Beenker, F.: Eindhoven University of Technology, TH-Report 82-WSK-04, Sept. 1982

3. Duneau, M., Katz, A.: J. Phys. 47, 181 (1986)

4. Gahler, F., Rhyner, J.: J. Phys. A 19, 267 (1986)

5. Khintchin, A.Ya.: Continued fractions. New York: Dover 1967

Communicated by Ya. G. Sinai

Received April 20, 1988 
